This paper is published in the open archive of Mid Sweden University

DIVA http://miun.diva-portal.org

by permission of the publisher

Sylvain Tourancheau, Kun Wang, Jarosław Bułat, Romain Cousseau, Lucjan Janowski, Kjell Brunnström and Marcus Barkowsky, "Reproducibility of crosstalk measurements on active glasses 3D LCD displays based on temporal characterization", Proc. SPIE 8288, 82880Y (2012);

http://dx.doi.org/10.1117/12.908082

(C) Copyright 2012 Society of Photo-Optical Instrumentation Engineers. One print or electronic copy may be made for personal use only. Systematic electronic or print reproduction and distribution, duplication of any material in this paper for a fee or for commercial purposes, or modification of the content of the paper are prohibited. 


\title{
Reproducibility of crosstalk measurements on active glasses 3D LCD displays based on temporal characterization
}

\author{
Sylvain Tourancheau ${ }^{* a}$, Kun Wang ${ }^{a, b}$, Jarosław Bułat ${ }^{c}$, Romain Cousseau ${ }^{d}$, \\ Lucjan Janowski ${ }^{c}$, Kjell Brunnström ${ }^{b}$, Marcus Barkowsky ${ }^{d}$ \\ ${ }^{a}$ Dept. of Information Technology and Media, Mid Sweden University, Sundsvall, Sweden \\ ${ }^{b}$ NetLab: IPTV, Video and Display Quality, Acreo AB, Kista, Sweden \\ ${ }^{c}$ Dept. of Telecommunications, AGH University of Science and Technology, Kraków, Poland \\ ${ }^{d}$ IRCCyN (UMR CNRS 6597), Polytech'Nantes, University of Nantes, Nantes, France
}

\begin{abstract}
Crosstalk is one of the main display-related perceptual factors degrading image quality and causing visual discomfort on 3D-displays. It causes visual artifacts such as ghosting effects, blurring, and lack of color fidelity which are considerably annoying and can lead to difficulties to fuse stereoscopic images. On stereoscopic LCD with shutter-glasses, crosstalk is mainly due to dynamic temporal aspects: imprecise target luminance (highly dependent on the combination of left-view and right-view pixel color values in disparity regions) and synchronization issues between shutter-glasses and LCD. These different factors influence largely the reproducibility of crosstalk measurements across laboratories and need to be evaluated in several different locations involving similar and differing conditions. In this paper we propose a fast and reproducible measurement procedure for crosstalk based on high-frequency temporal measurements of both display and shutter responses. It permits to fully characterize crosstalk for any right/left color combination and at any spatial position on the screen. Such a reliable objective crosstalk measurement method at several spatial positions is considered a mandatory prerequisite for evaluating the perceptual influence of crosstalk in further subjective studies.
\end{abstract}

Keywords: crosstalk, temporal measurements, stereoscopic displays, shutter-glasses, time-sequential

\section{INTRODUCTION}

Crosstalk is one of the main display-related perceptual factors degrading image quality and causing visual discomfort on 3D-displays. ${ }^{1,2,3}$ It is usually defined as "the incomplete isolation of the left and right image channels so that one image leaks into the other". ${ }^{4}$ It can be due to many factors varying according to $3 \mathrm{D}$ display technologies $^{5}$ and many definitions and terms have been proposed in literature over the past twenty years. ${ }^{4}$ Crosstalk manifests itself through visual artifacts such as ghosting, blurring, and lack of color fidelity ${ }^{6}$ and provokes general annoyance and visual discomfort. Depth perception can be affected as well; to some extent crosstalk can even lead to stereoscopic depth breakdown. ${ }^{7}$ Figure 1 illustrates ghosting for a simple stereoscopic pair. The "leaking" of the right image in the left view and vice versa provokes the apparition of a double image. This phenomenon is particularly noticeable and annoying in disparity regions, i.e. when right and left images have different values.

One of the current projects of the Video Quality Expert Group (VQEG) ${ }^{8}$ is to standardize the viewing environment for 3D presentations. In this context, VQEG plans to perform a multi-laboratory evaluation of the objective and subjective measurement of crosstalk on stereoscopic displays. A primary goal of this project is to propose a fast and simple method of crosstalk measurement that provides reproducible results across different environments and laboratories. This paper present the results of a campaign of objective crosstalk measurements carried out in three different laboratories and involving similar and differing conditions. This campaign has been focused on time-sequential 3D liquid crystal displays (LCDs) using active shutter-glasses.

The rest of this document is organized as follows: Section 2 describes how crosstalk manifest itself on timesequential stereoscopic LCDs and reviews existing crosstalk metrics in that context. Sections 3 presents the two measurements procedures used in this study and the various test conditions involved. Section 4 presents the results of the measurements. Finally Section 5 discusses the obtained results and conclude the main points of the work.

* Corresponding author: sylvain.tourancheau@miun.se 

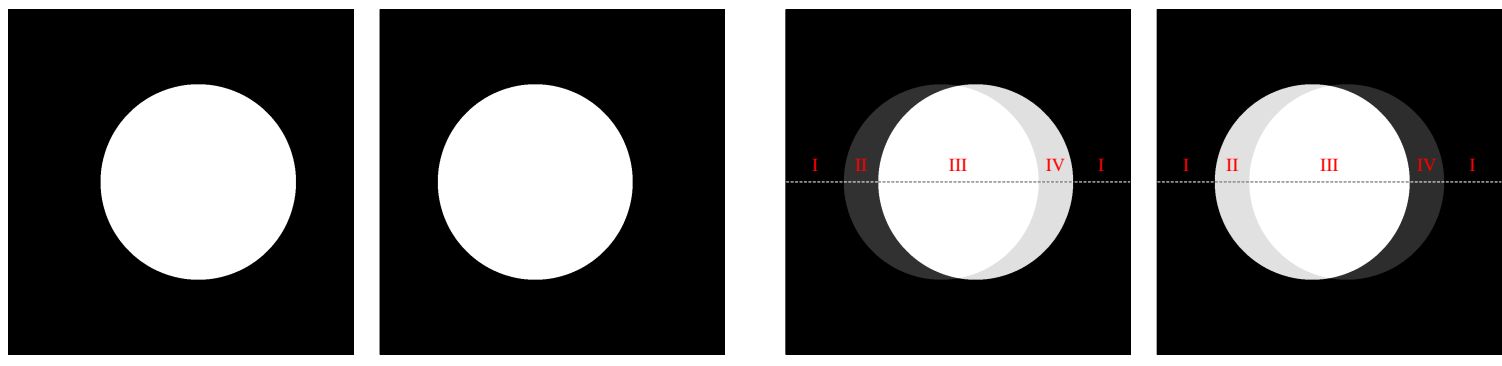

(a) Left and right images, inputs of the stereoscopic dis-(b) Left and right views, outputs of the stereoscopic display. play.

Figure 1: Illustration of ghosting with a simple stereoscopic pair of images. (a) Left and right digital images sent to the stereoscopic visualization system. (b) Left and right views as measured through the complete visualization system (e.g. display and glasses if any). Four regions can be defined in that case. Regions I and III are the zones where right and left signal have the same value, while zones II and IV are the disparity regions where right and left signals are different.

\section{CROSSTALK ON TIME-SEQUENTIAL STEREOSCOPIC LCDS}

There are so far no standardized procedures concerning crosstalk measurements and crosstalk metric calculations. For two-view stereoscopic visualization systems, the crosstalk ratio is usually defined with respect to black and white input signal. A definition commonly used ${ }^{4,5,9}$ is:

$$
C^{L}=\frac{\bar{L}_{0,255}^{L}-\bar{L}_{0,0}^{L}}{\bar{L}_{255,0}^{L}-\bar{L}_{0,0}^{L}}, \quad C^{R}=\frac{\bar{L}_{255,0}^{R}-\bar{L}_{0,0}^{R}}{\bar{L}_{0,255}^{R}-\bar{L}_{0,0}^{R}}
$$

where $C^{L}$ is the crosstalk in the left channel, defined as the ratio between the average luminance $\bar{L}_{0,255}^{L}$, measured from left eye position when left input image is a full-black image (all pixels value is 0 ) and right input image is a full-white image (all pixels value is 255), and the average luminance $\bar{L}_{255,0}^{L}$, measured from left eye position when left input image is a full-white image and right input image is a full-black image. Usually, but not always, ${ }^{4}$ the non-zero black level of stereoscopic displays is taken into consideration by subtracting the black level average luminance $\bar{L}_{0,0}^{L}$ (i.e. the average luminance measured from left eye position when both left and right input image are full black images). The same definition applies for crosstalk in the right channel $C^{R}$, with average luminance values measured from the right eye position.

Since crosstalk can be considered as an additive and linear phenomenon in most 3 D-displays, ${ }^{4}$ the use of full-white and full-black images permits to measure conditions for which maximum crosstalk usually occurs and therefore provides a good estimation of display's overall crosstalk. However, this is not true anymore for displays that exhibit non-linear and non-additive crosstalk. In particular on time-sequential stereoscopic liquid-crystal displays (LCDs) using active shutter-glasses, crosstalk is mainly due to temporal characteristics (response time and synchronization) of the system's components (display and active shutter-glasses). ${ }^{10,11}$

More precisely, the luminance emitted by pixels within a frame period is usually imprecise because of the slow response of the liquid crystal cells, or because of hardly controllable response-time reduction systems (which lead to luminance over- or under-shoots). This issue is hardly predictable and depends on the combination of left-view and right-view pixel color values in disparity regions. ${ }^{12}$ Another cause of crosstalk comes from the synchronization between shutter-glasses and LCD: the shutter open-period cannot be equally synchronized with the whole display because of the temporal delay between first and last lines update. Displays enhancement functions such as back-light flashing can even make this problem worse. ${ }^{13}$

Figure 2 illustrates how and why ghosting artifacts appear on time-sequential 3D-LCDs, using for this purpose the simple example presented in Figure 1. Four different regions are defined: regions I and III are the zones 
where right and left images have the same value, while zones II and IV are the disparity regions where right and left images are different. Figure 2a illustrates the display luminance for each of this four regions. In zone I (resp. zone III), both left and right images have the same value $i$ (resp. $j$ ), the luminance measured on the display $L_{i, i}^{D}(t)$ (resp. $\left.L_{j, j}^{D}(t)\right)$ in then a steady signal. In zone II (resp. zone IV), left and right image have different values and the luminance emitted by the display $L_{i, j}^{D}(t)$ (resp. $L_{j, i}^{D}(t)$ ) alternates between $i$ and $j$ (resp. between $j$ and $i$ ).

On time-sequential 3D-LCDs, right and left images are separated and guided to corresponding eyes thanks to active shutter-glasses synchronized with the display in order to be open when the corresponding signal is displayed on the screen. Figure 2c illustrates the temporal transmittance functions $\tau_{L}(t)$ and $\tau_{R}(t)$ of each shutter-glass. These transmittance functions are synchronized with the display luminance waveforms presented in Figure 2a. The luminance signal seen through each glass is then the result of the multiplication between the transmittance function of each shutter-glass and the display luminance, for example in region II:

$$
\begin{aligned}
& L_{i, j}^{L}(t)=\tau_{L}(t) \cdot L_{i, j}^{D}(t) \\
& L_{i, j}^{R}(t)=\tau_{R}(t) \cdot L_{i, j}^{D}(t)
\end{aligned}
$$

The resulting luminance waveforms measured through left glass $L_{i, j}^{L}(t)$ and right glass $L_{i, j}^{R}(t)$, in region II, are plotted in Figure2b and Figure2d, along with those corresponding to the other regions.

Finally, the average luminance level of region II as seen from each eye position is obtain by averaging the previous waveforms over a whole number of periods:

$$
\begin{aligned}
\bar{L}_{i, j}^{L} & =\int_{t_{0}}^{t_{0}+N \cdot T} L_{i, j}^{L}(t) \cdot d t \\
\bar{L}_{i, j}^{R} & =\int_{t_{0}}^{t_{0}+N \cdot T} L_{i, j}^{R}(t) \cdot d t
\end{aligned} \quad N \in \mathbb{N}
$$

Average luminance levels measured from each eye position for each region of the stereoscopic image are presented as a function of the horizontal axis in Figure 3. It can be observed that these luminance levels are not necessarily identical from one eye position to the other due to slightly asymmetrical transmittance functions.

It is clear from this simple example that the ghosting occurring in time-sequential 3D-LCDs is mainly due to the failure of the display to reach the correct luminance level within the short frame period ( $8.33 \mathrm{~ms}$ for $120-\mathrm{Hz}$ displays). Since the response time of display pixels depends highly on the starting and ending levels of luminance, the crosstalk metric given by Equation 1 - which concerns only the black and white combination - cannot be representative of the overall crosstalk of the display. For this reason, several crosstalk metrics have been recently proposed in the literature. ${ }^{12,13,14,15,16}$ They propose to compute the crosstalk ratio for any combination $\{i, j\}$ of left and right pixels values. These color values are usually grey values, i.e. each sub-pixels is submitted to the same digital input: $R=G=B=i$ and $R=G=B=j$. The crosstalk ratio is often referred to grey-to-grey crosstalk in that case. These crosstalk metrics are given here for a left-eye position, and original expressions have been adapted to follow the notations defined previously in this paper (average luminance levels depicted in Figure 3).

Pan et al. ${ }^{13}$ and Shestak et al. ${ }^{12}$ proposed two very similar definitions which consist of the ratio between the error of luminance and the amplitude of the considered grey-to-grey transition. The equations are respectively:

$$
\begin{gathered}
C_{i, j}^{L}=\frac{\left|\bar{L}_{i, i}^{L}-\bar{L}_{i, j}^{L}\right|}{\bar{L}_{i, i}^{L}-\bar{L}_{j, j}^{L}} \\
C_{i, j}^{L}=\frac{\bar{L}_{i, j}^{L}-\bar{L}_{i, i}^{L}}{\bar{L}_{j, j}^{L}-\bar{L}_{i, i}^{L}}
\end{gathered}
$$




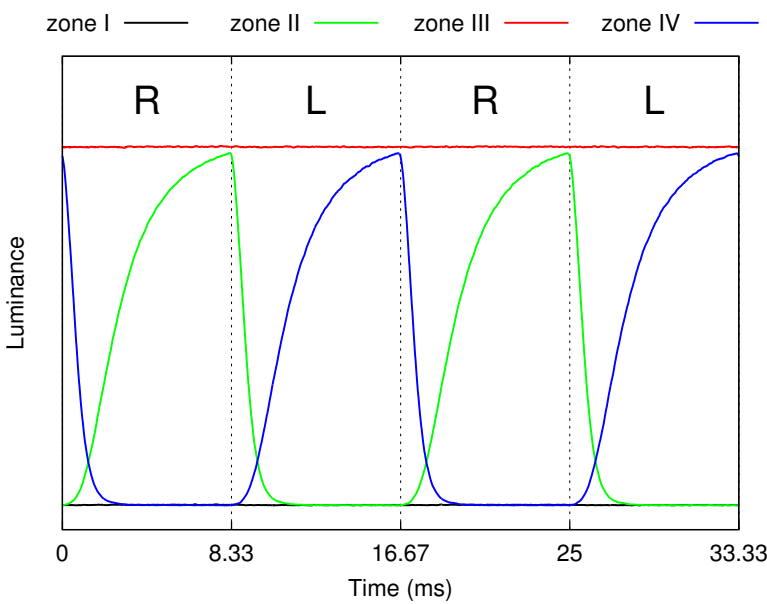

(a) Luminance measured directly on the display.

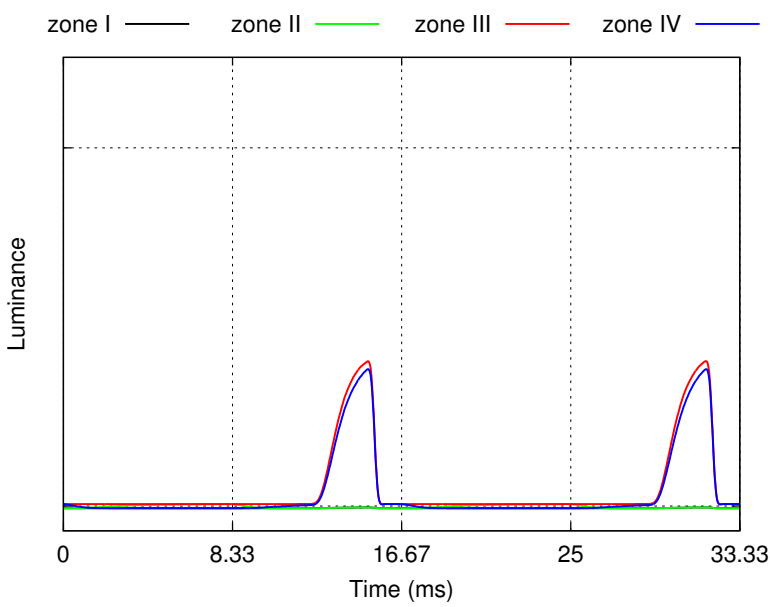

(c) Luminance measured through the left glass.

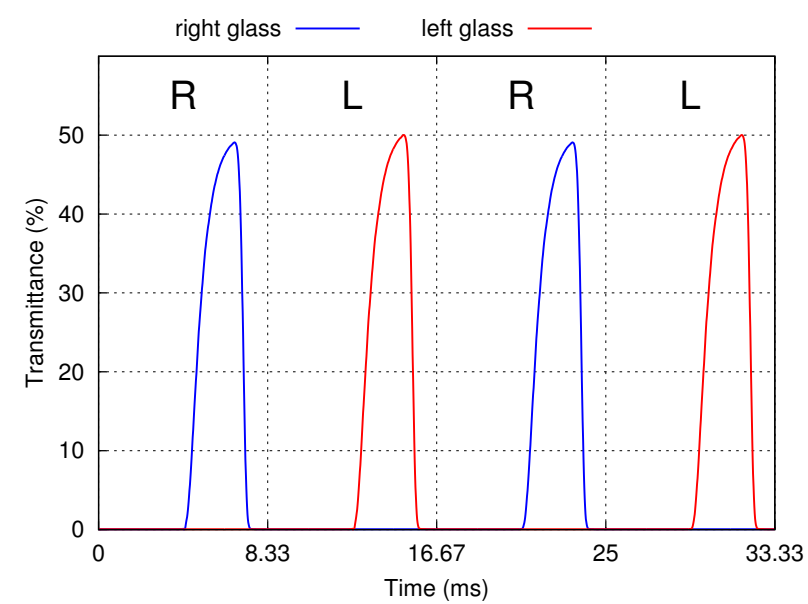

(b) Temporal transmittance of right and left shutter-glasses.

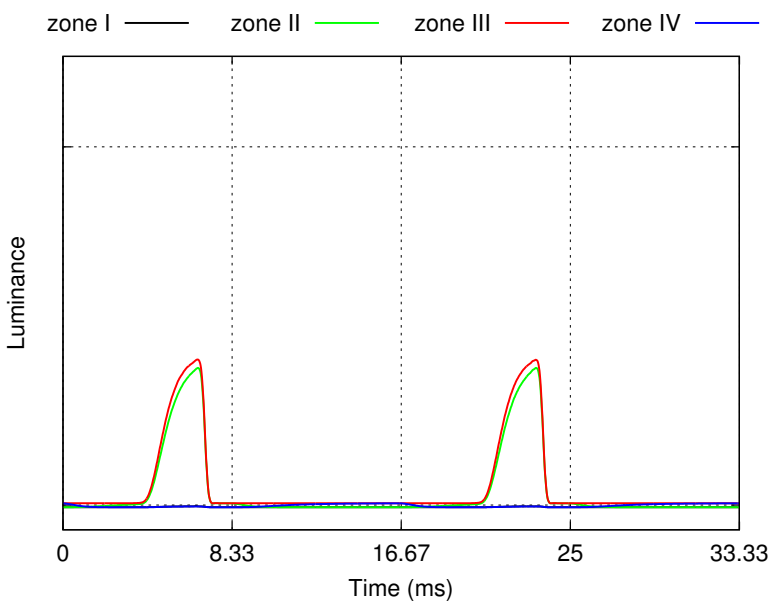

(d) Luminance measured through the right glass.

Figure 2: Luminance signals in the four regions defined in Figure 1. (a) Luminance measured directly on the display, the alternation between right and left values can be observed for regions II and IV (disparity regions). (b) Transmittance of the shutter-glasses as a function of time, synchronized with the display signal. (c-d) Luminance measured through the left and right shutter-glass respectively, the waveforms corresponds to the display luminance signals multiplied by the shutter-glass transmittance (cf. Equation 2). 


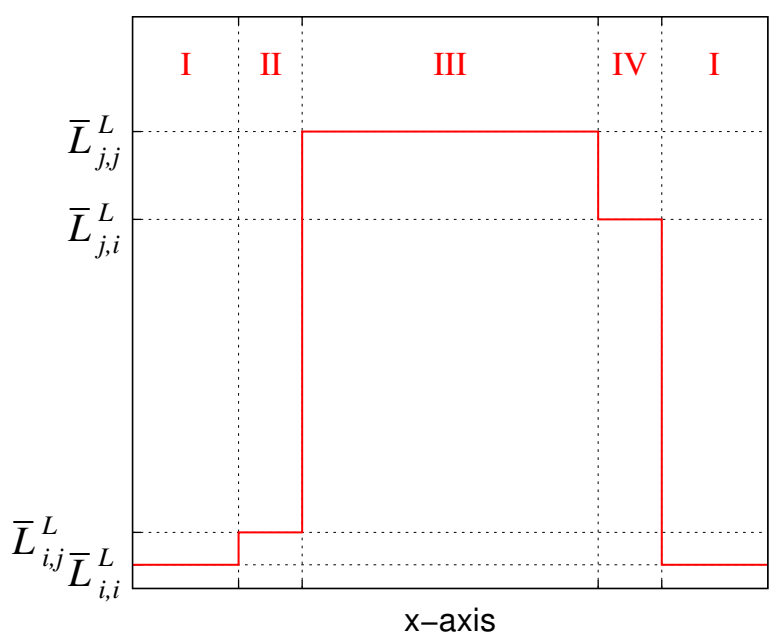

(a) Left view.

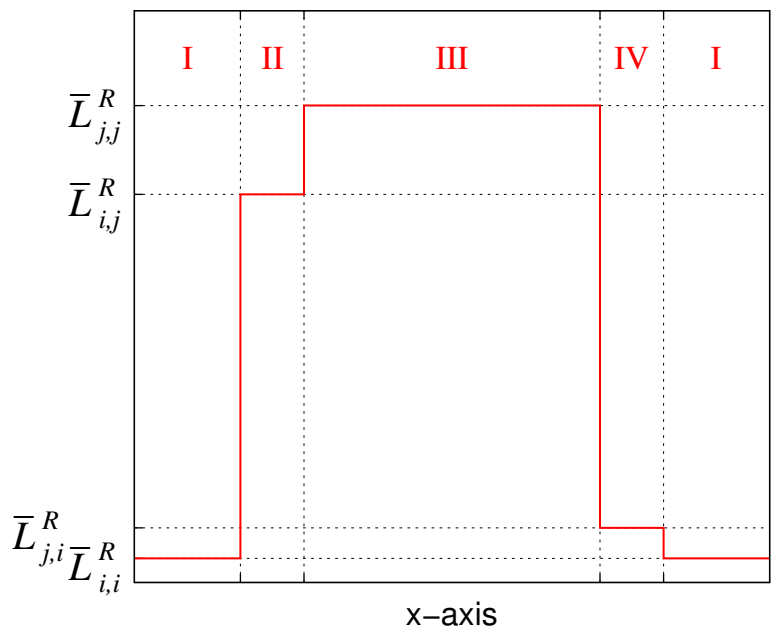

(b) Right view.

Figure 3: Spatial profiles observed from the left eye position (a) and from the right eye position (b) along the dotted line in Figure 1. Values correspond to the average luminance measured through each glass for each regions of the stereoscopic image (cf. Equation 3).

Jung et al. ${ }^{14}$ proposed a similar definition, directly inspired from the black-white crosstalk defined in Equation 1. The difference with the two previous definitions comes from the denominator which in that case take into account the opposite combination (just as Equation 1 does):

$$
C_{i, j}^{L}=\left|\frac{\bar{L}_{i, j}^{L}-\bar{L}_{i, i}^{L}}{\bar{L}_{j, i}^{L}-\bar{L}_{i, i}^{L}}\right|
$$

Finally, Bułat et al. ${ }^{16}$ defined grey-to-grey crosstalk directly as the relative error of luminance:

$$
C_{i, j}^{L}=\frac{\left|\bar{L}_{i, j}^{L}-\bar{L}_{i, i}^{L}\right|}{\bar{L}_{i, i}^{L}}
$$

It has been chosen here to keep using the term crosstalk as in previous literature even if in the case of time-sequential 3D-LCDs the main cause of ghosting is not a luminance leakage from one channel to another (as crosstalk is usually defined) but rather a failure of the display to reach correct luminance levels with respect to different conditions.

\section{MEASUREMENTS}

\subsection{Conditions}

As it has been shown previously, crosstalk on time-sequential 3D-LCDs is mainly due to the slow response time of the display when the luminance has to alternate between right and left value, and therefore is highly dependent on the right-left combination of pixels values. In order to figure out how important are these crosstalk variations, it has been decided to conduct a fully comprehensive measurement campaign, testing all 65536 grey-to-grey combinations of the display. Moreover, these measurements have been performed at three different positions on the screen in order to study the influence of the synchronism between the shutter-glasses and the display.

Measurements have been conducted in three different laboratories:

- Lab1: Realistic3D Lab, Mid Sweden University (affiliation $a$ on the first page) 
- Lab2: NetLab, Acreo AB (affiliation b)

- Lab3: IVC Lab, IRCCyN (affiliation $d$ )

Two different measurement methods have been used in this study:

1. The first method consists in measuring luminance through the complete stereoscopic system (display and shutter-glasses) for different grey-to-grey combinations.

2. The second one consists in measuring the grey-to-grey temporal transitions of the display alone and then applying the transmittance functions of the shutter-glasses to obtain the luminance as seen from each eye-position (cf. Equation 2).

The first method presents the inconvenient to be very sensitive to the measurement's setup, to the position and orientation of the glasses, the measured field of view, etc. To properly handle these parameters, complex optical arrangements are necessary (e.g. Fabry assemblies ${ }^{17}$ ) which are usually expensive and necessitate a complex expertise. Such a protocol does not fit the current VQEG requirements, that is to say to propose a fast and simple measurement protocol which could be easily carried out in various labs. The second method presents the advantage of requiring only close-contact measurements on the display: this kind of measurements is usually easier to carry out and less subject to variation from lab to lab. This method is similar to the one presented by Boher et al. ${ }^{11}$ except that their study used simulated display transitions for combinations of 9 grey levels while the work presented here uses real display measurements of all 65536 grey-to-grey combinations.

\subsection{Equipment}

\subsubsection{Photo-diodes}

Due to the dynamic nature of the problem, the measurement methods used in this study consist of high samplerate measurements of the temporal responses of both shutter-glasses and displays. For the reasons mentioned above, some close-contact instruments, that do not necessitate any optics assemblies, have been used. The three instruments are quite comparable to each other and based on fast photo-diode electronic circuits. Details are given here:

- Lab1: Siemens BPW21 silicon photo-diode. Response time: $25 \mu \mathrm{s}$ for rise time, 41 us for fall time.

- Lab2 and Lab3: Burr-Brown OPT101 monolithic photo-diode. Response time: 28 us for rise and fall times.

Photo-diodes are housed in boxes to shield any ambient light and surrounded by black velvet in order to avoid any scratches to the display surface. Signals are captured by a dual-channel 12-bit USB oscilloscope (DS1M12 from EasySync Ltd.) with a sampling period of $20 \mu \mathrm{s}$.

\subsubsection{Conversion to luminance}

Voltage signals obtained from the photo-diodes are then converted into luminance values thanks to the following luminance-meters:

- Lab1: KonicaMinolta LS-110

- Lab2: Photo Research PR522/524

- Lab3: CRS Optical OP200-E 


\begin{tabular}{|c|c|c|c|c|c|c|c|c|c|c|c|c|}
\hline & \multicolumn{5}{|c|}{ Left glass } & \multicolumn{5}{|c|}{ Right glass } & & \\
\hline & $\# 1$ & $\# 2$ & $\# 3$ & $\# 4$ & $\# 5$ & $\# 1$ & $\# 2$ & $\# 3$ & $\# 4$ & $\# 5$ & Average & Std Dev \\
\hline Average trans. & 6.16 & 6.12 & 5.90 & 5.98 & 5.95 & 5.71 & 5.97 & 5.50 & 5.66 & 5.40 & 5.84 & 0.257 \\
\hline Maximum trans. & 49.1 & 49.8 & 50.4 & 48.8 & 49.1 & 48.2 & 50.1 & 49.0 & 47.9 & 47.0 & 48.9 & 1.03 \\
\hline
\end{tabular}

Table 1: Average and maximum transmittance of five different pairs of shutter-glasses.

Conversion functions have been determined independently in each lab by measuring the steady luminance value of each of the 256 grey levels simultaneously with the photo-diode and the luminance-meter. These measures have been acquired in the center of the display, with and without shutter-glasses. Characteristics of the photodiodes have been found quite linear as expected and intermediate values have been interpolated to build three look-up-tables used to convert raw data from each lab. However, it must be mentioned here that the validity and the accuracy of this conversion is questioned for luminance values below $0.1 \mathrm{~cd} / \mathrm{m}^{2}$. Firstly because the luminance-meters that have been used are not accurate anymore in this range, and secondly because of the influence of the dark offset of photo-diodes.

In order to facilitate comparison between each lab, all results have been normalized with respect to the average luminance of the display measured in the center position for a combination of right and left full-white images (i.e. $\bar{L}_{255,255}^{D}$ ). This permits to reduce possible variations due to the differences in the calibration of the three luminance-meters, and to the differences of maximum luminance of the three display samples.

\subsubsection{Stereoscopic system under test}

Three different samples of Alienware display OptX AW2310 display have been measured. They have been used in their native resolution $(1920 \times 1080$ pixels $)$ with a refresh rate of $120 \mathrm{~Hz}$, together with $\mathrm{PC}$ equipped with NVIDIA 3D vision system (active liquid-crystal shutter-glasses). Stereoscopic images have been presented with Matlab and the PsychToolbox. ${ }^{18}$

Display internal settings have been set identically in the three labs, and display have been turned on few hours before each measurements session.

\subsection{Shutter-glasses temporal characterization}

\subsubsection{Transmittance of shutter-glasses}

To study the variability of the NVIDIA LC shutter-glasses used in this work, the transmittance functions of five different shutter-glasses have been chracterized in Lab1. The luminance emitted by a white LED source have been measured through each glass of the five pairs. During these measurements, the photo-diode, the glass and the LED source have been aligned as close to each other as possible. The small size of the LED source permitted to keep a narrow field of view. Since these shutter-glasses are made of liquid crystal, their transmittance depends on the polarization of the light. The light source used in these measurements was non-polarized, while the light emitted by a LCD is polarized by definition. In order to obtained transmittance values which correspond to the transmittance observed when the active glasses are used with the display under test, the waveforms have been scaled according the luminance ratios measured through the shutter-glasses with luminance-meters. The same unique scaling function have been used for all pairs of shutter-glasses in order to keep the variability between samples. Each glass have been measured during 350 frames, and the waveforms corresponding to each period have been averaged together to reduce noise and potential variations. Figure 4 presents the transmittance of the right glass of each of the five pairs as a function of time with a logarithmic scale. It can be observed that the full width at the half height is about $2 \mathrm{~ms}$, with an opening time of approximately $1.5 \mathrm{~ms}$ and a closing time of approximately $0.3 \mathrm{~ms}$. These results are in concordance with those presented by Boher et al. ${ }^{11}$ Table 1 summarizes the maximum and average transmittance for each glass of the five pairs of shutter. The average transmittance is about $5.8 \%$ (i.e. maximum luminance seen through one glass is about $20 \mathrm{~cd} / \mathrm{m}^{2}$ for the display under test which has a maximum luminance of about $350 \mathrm{~cd} / \mathrm{m}^{2}$ ), with a standard deviation of $0.26 \%$. 


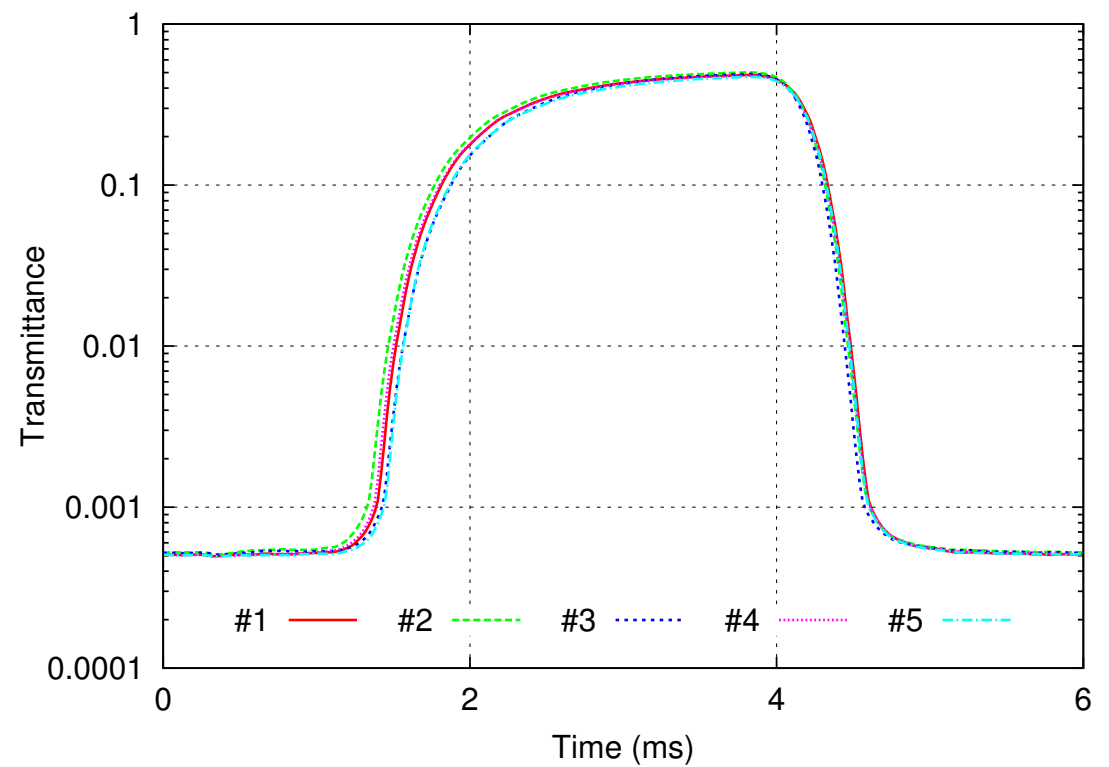

Figure 4: Comparison of the transmittance of five shutter-glasses (right-glass). The differences are summarized in Table 1.

\subsubsection{Synchronization between the display and the shutter-glasses}

In order to know the synchronism between display and shutter-glasses, the synchronization signal sent to the glasses by the infra-red emitter of the NVIDIA 3D Vision system has been measured. This sync signal has been acquired directly across the infra-red LEDs inside the emitter. Figure 5 illustrates the synchronism between this sync signal and the transmittance of the pair of shutter-glasses \#1. In the following, the sync signal is measured simultaneously to all display measurements, in order to determine the synchronization between the display waveforms and the transmittance functions. Because of the top-to-bottom vertical scanning of LCDs, the opening period of the shutter-glasses is not equally synchronized with the display temporal responses. Figure 6 illustrates this difference for the three different measuring spots on the screen.

\subsection{High-sample rate measurements}

First method: display + shutter-glasses The first method have been carried out only in Lab3. Measurements have been performed through the right glass for all combinations between 129 grey levels (from 0 to 254 with a step of 2, plus 255) in the center position, and for all combinations between 65 grey levels (from 0 to 252 with a step of 4, plus 255) in the top-left and bottom-right positions. A specific set-up has been designed in order to perform those measurements with a contact instrument: acquisition has been performed with the display lying flat, the photo-diode, the shutter-glass, and the display were aligned as close together as possible. Two small rings of non-reflective synthetic black foam rubber were disposed between the photo-diode and the shutter-glass and between the shutter-glass and the display in order to maintain a narrow measured field of view.

Second method: display alone + shutter-glasses simulation The second method have been carried out in the three labs. All right and left combinations of the 256 grey levels have been measured in Lab1 and Lab2, for the three positions on the screen. Combinations between 65 grey levels (from 0 to 252 with a step of 4, plus 255) have been measured in Lab3, for the three positions on the screen. For each grey-to-grey combination, the synchronization signal have been acquired simultaneously. This synchronization signal has been afterward compared to the reference sync signal measured previously (cf. Section 3.3.2) in order to synchronize the transmittance functions of right and left shutter-glasses with the display temporal responses and therefore obtain the luminance values as seen from each eye position by the use of Equation 2. 


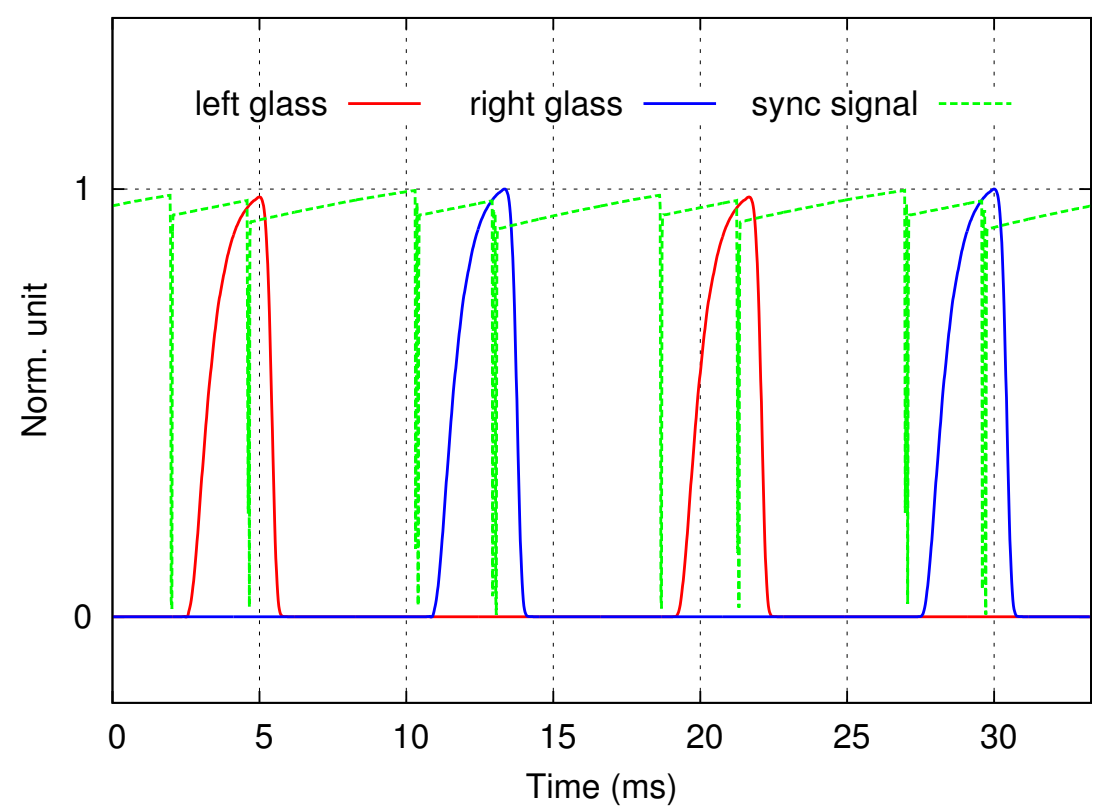

Figure 5: Transmittance of left and right shutter-glasses and synchronization signal measured directly in the emitter across IR LEDs. Signals have been arbitrarily normalized between 0 and 1 .

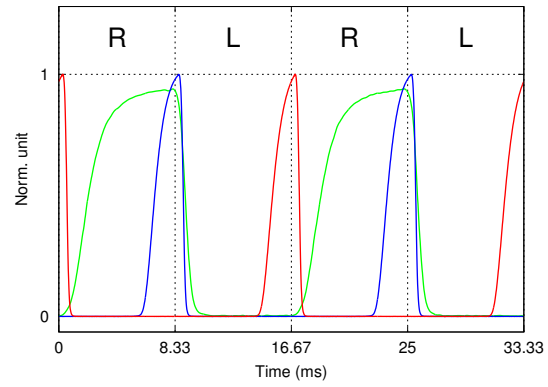

(a) Top-left position

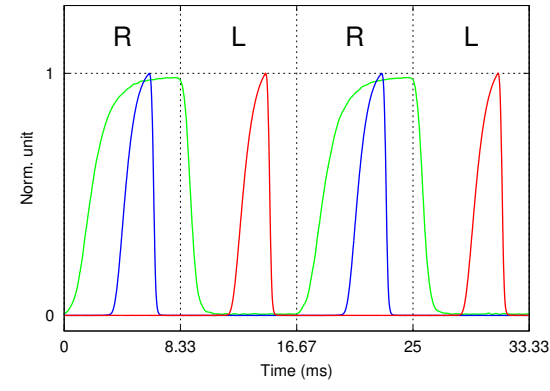

(b) Center position

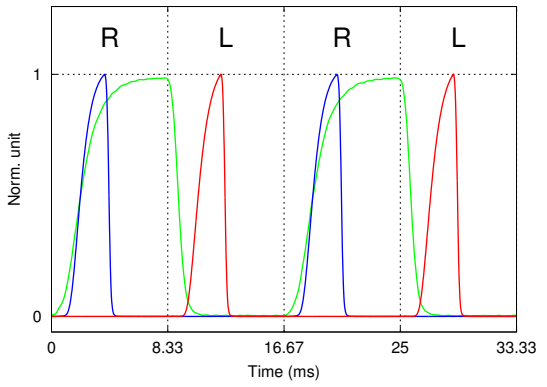

(c) Bottom-right position

Figure 6: Synchronization between display and shutter-glasses for different positions on the screen. Waveforms have been measured for a combination $i=0$ and $j=255$, in the top-left position (a), in the center of the screen (b), and in the bottom-right position (c). Luminance of the display has been normalized with respect to the white luminance (measured for a combination $i=j=255$ ). Transmittance functions of the shutter-glasses have been normalized between 0 and 1 for clarity. 

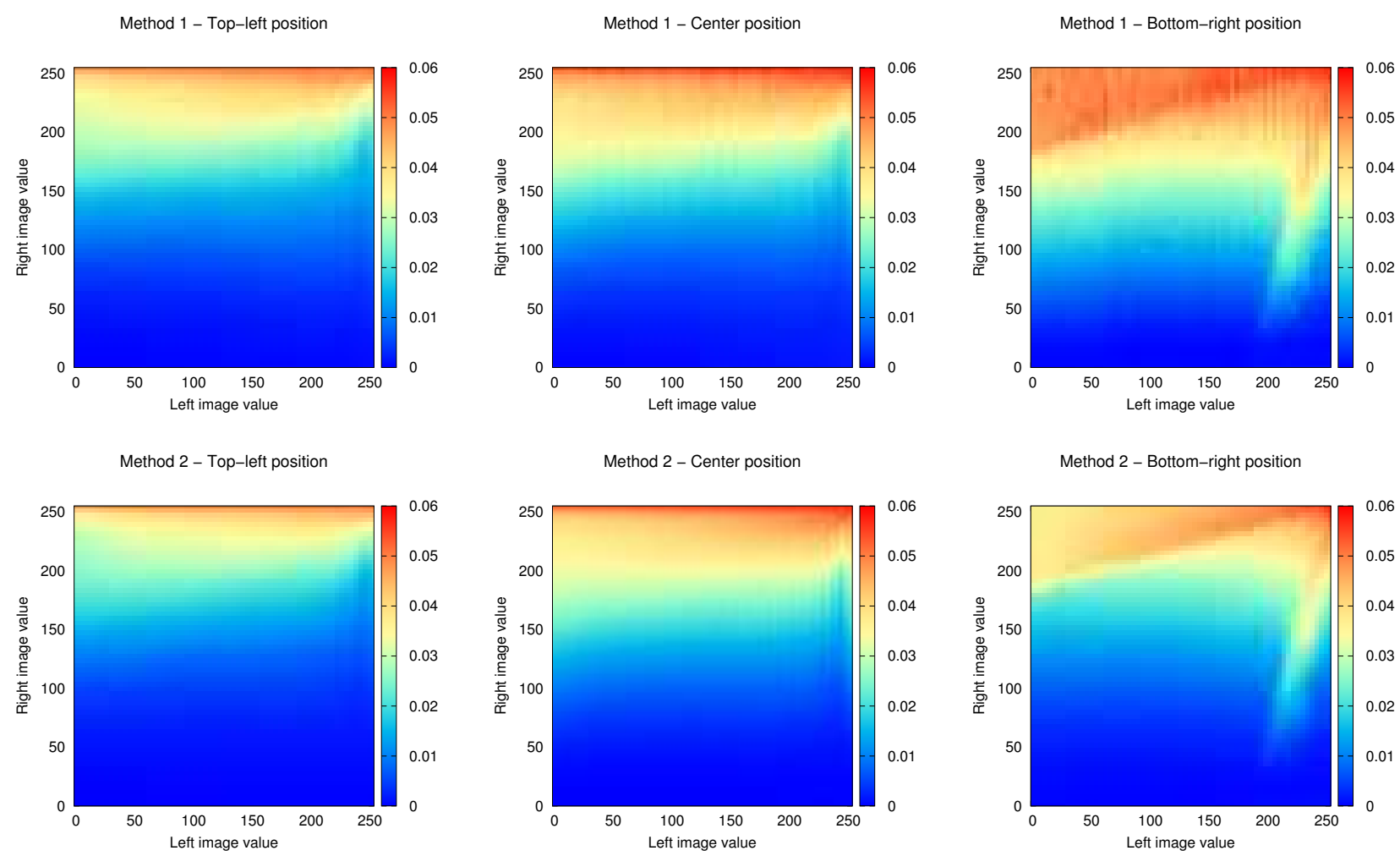

Figure 7: Comparison of both measurement methods at three positions on the screen. Top row shows luminance measured with the first method at the right-eye position for three different positions on the screen. Bottom row shows luminance values obtained from the second method, i.e. by the multiplication of right-glass transmittance with luminance waveforms measured directly on the display.

For both methods, the final luminance waveforms (directly measured through the shutter-glass, or obtained by a simulation of the shutter-glass transmittance) are finally averaged over a whole number of periods to obtain the mean luminance as measured from each eye position (Equation 3). Each grey-to-grey transition has been measured for a duration of $400 \mathrm{~ms}$ ( 48 frames, 24 frames for each view), i.e. 20000 samples. In average, it took around 1 second to measure one combination (overhead comes from data saving, oscilloscope commands sending and receiving, etc.) meaning that one complete measurement session took around 18 hours to measure the 65536 combinations at one position on the screen.

\section{RESULTS}

\subsection{Luminance}

Final measurements results are obtained under the form of matrices presenting the luminance values seen from one eye-position for any combination $\{i, j\}$ of left and right values. Right values are varying horizontally and left values are varying vertically. If the stereoscopic visualization system was not suffering from crosstalk, all lines of the right-eye luminance matrices would be constant (no influence of the left value on the luminance corresponding to one given right value) and consequently all columns of the matrix would be similar. Similarly, on a perfect system the left-eye luminance matrices would have constant columns.

Figure 7 presents the luminance values measured at the right-eye position with both methods. It can be first observed that the results from both methods are very similar, particularly on top-left and center positions (linear correlation coefficients of 0.9959 and 0.9989 respectively). For bottom-right position, differences between both methods are more noticeable but the linear correlation coefficient is still high (0.9323). This difference might 
Lab 1 - Top-left position - Left-eye

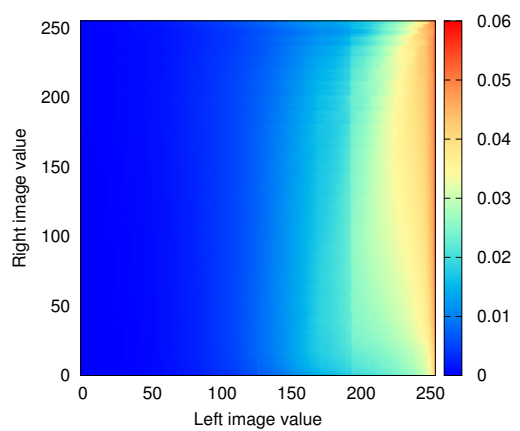

Lab 2 - Top-left position - Left-eye

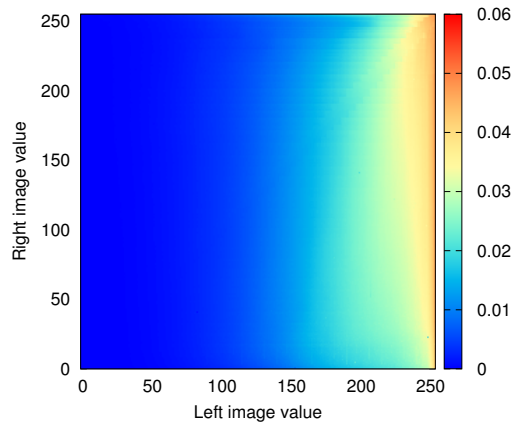

Lab 3 - Top-left position - Left-eye

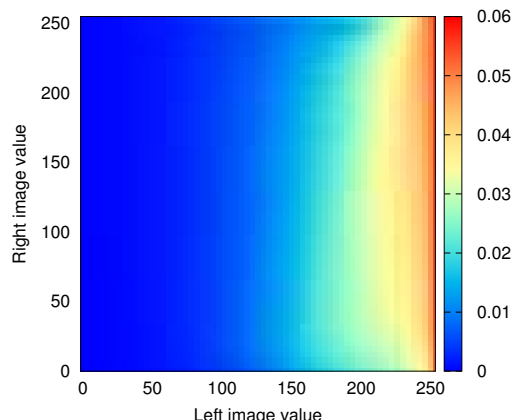

Lab 1 - Center position - Left-eye

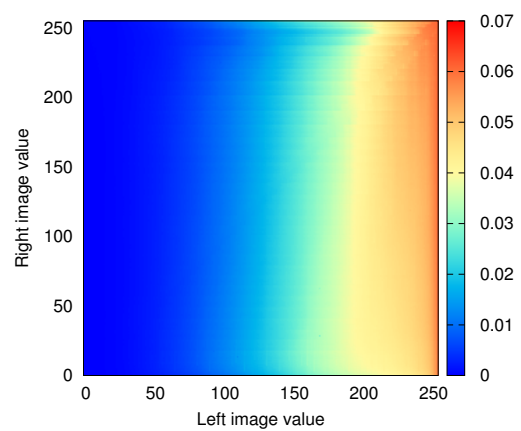

Lab 2 - Center position - Left-eye

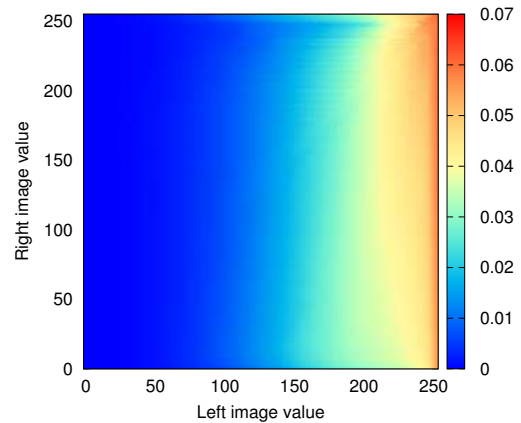

Lab 3 - Center position - Left-eye

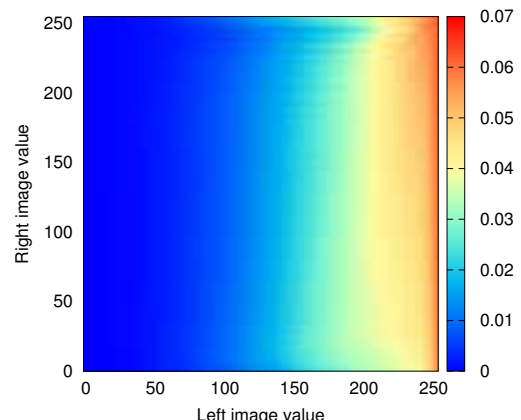

Lab 1 - Bottom-right position - Left-eye

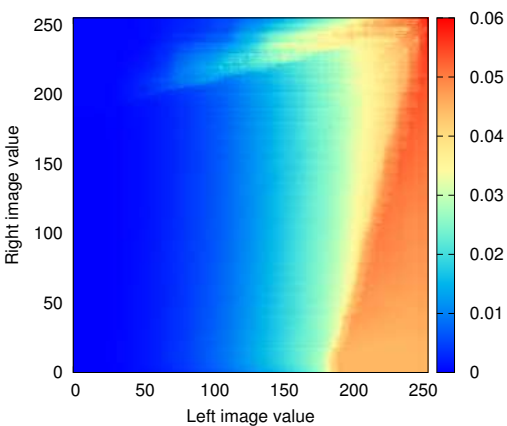

Lab 2 - Bottom-right position - Left-eye

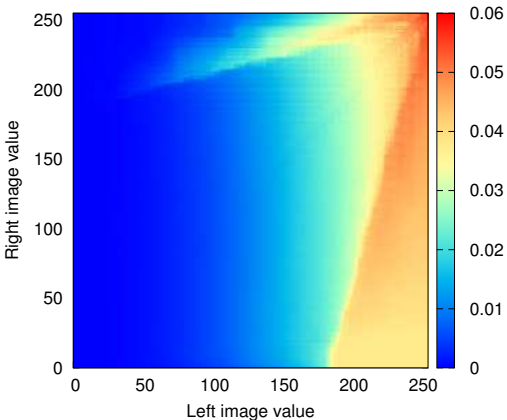

Lab 3 - Bottom-right position - Left-eye

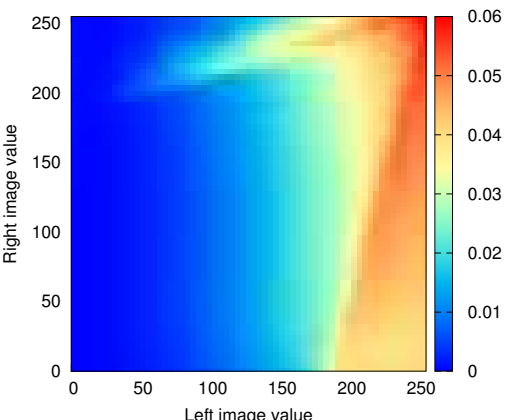

Figure 8: Luminance measured at the left-eye position with the second method in the three different labs, for three positions on the screen.

be due to a luminance conversion issue: the conversion functions which have been used to convert voltage into luminance have been calibrated according to measurements performed in the center of the screen. Despite these slight discrepancies, these first measurements give a good evidence that the second method - which consists in simulating the action of the shutter-glasses on temporal waveforms measured directly on the display - permits to obtain results very similar to those obtained by measuring through the shutter-glasses, while using a much simpler set-up.

Figure 8 presents the luminance values measured at the left-eye position with the second method only, for the three laboratories, and at three different positions on the screen. Luminance matrices are very similar from one lab to another and it is clear from these results that the position of the measuring spot on the screen has much more influence than the differences in terms of instruments or set-up from one lab to another. As expected, 

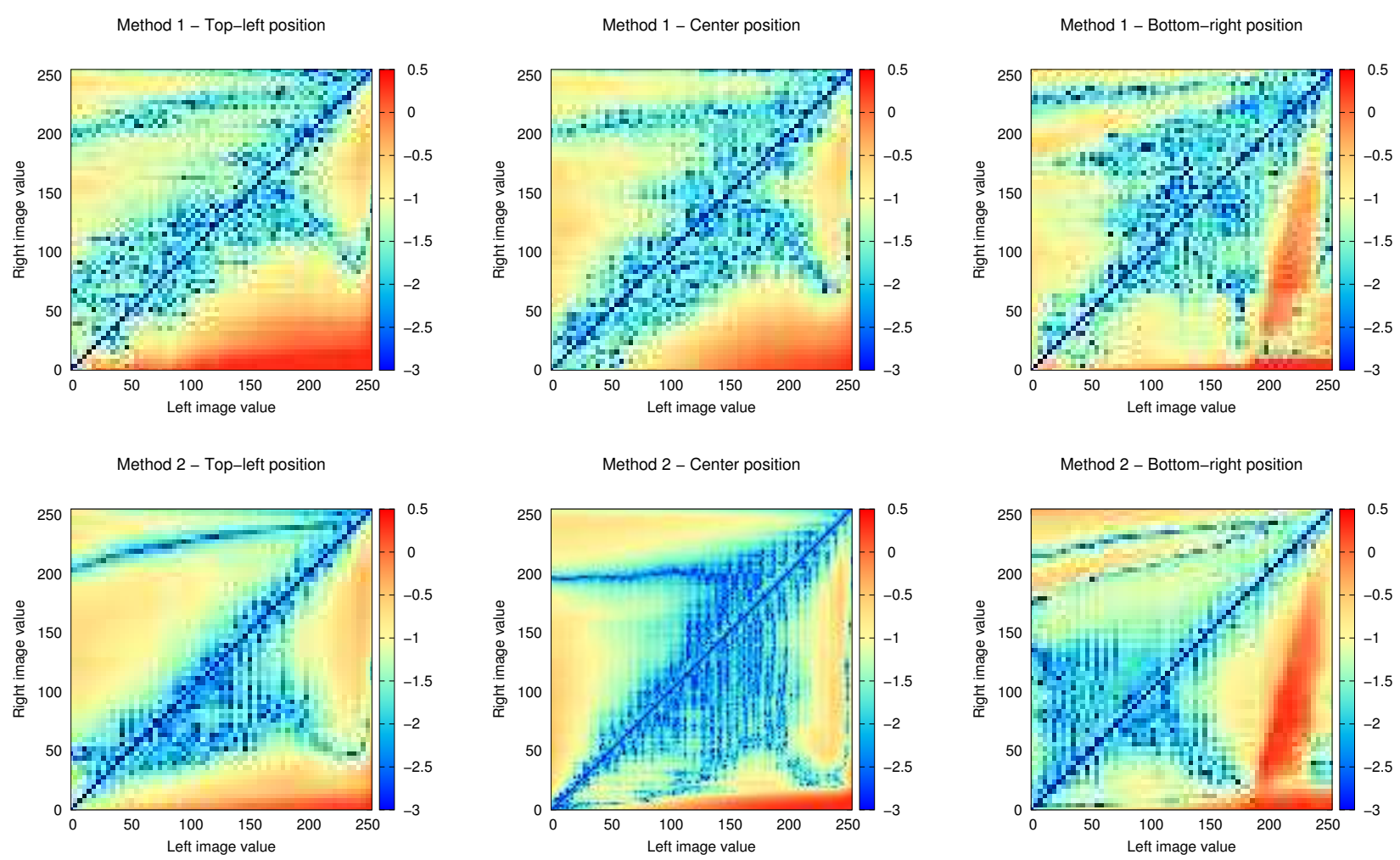

Figure 9: Crosstalk ratio at right-eye position (logarithmic scale) obtained with the first method (top row) and with the second method (bottom row), from the luminance matrices presented in Figure 7.

luminance matrices measured at the left eye position are roughly the transposes of those measured at the right eye position presented in Figure 7.

\subsection{Crosstalk ratio}

Crosstalk ratios for each eye position have been computed from the luminance matrices according to Equation 7 proposed by Bułat et al. ${ }^{16}$ The choice of this definition of grey-to-grey crosstalk among other ones has been mainly driven by the fact that it corresponds to a relative luminance error and is easily understandable. Furthermore, other definitions presents the inconvenient to have denominators which can be very close to zero when dealing with the whole grey-to-grey combinations and therefore can lead to crosstalk ratios tending to very high values. Again, crosstalk ratios for every combinations of right and left pixels value are presented under the form of matrices. For illustration and clarity purposes, the results in the figures are presented in a logarithmic scale, and values have been cropped between 0.001 and 3 (logarithmic values between -3 and 0.48 ), since for low grey levels in the measured view and high grey level in the other view, crosstalk ratios can be up to 10 (1000\%).

Figure 9 presents crosstalk ratios measured at the right-eye position in Lab3 with both measurement methods (computed from luminance values depicted in Figure 7), and Figure 10 presents crosstalk ratios at the lefteye position obtained from the second measurements method and compared across laboratories and measuring positions on the screen (computed from luminance values depicted in Figure 8). It can be observed from these figures that the crosstalk ratio is varying according to a pattern which is hardly predictable. Crosstalk evolution as a function of right and left pixels values is not monotonic and some "ridges" and "valleys" can be observed. However, despite this unpredictable pattern, crosstalk matrices measured at similar position of the screen are very recognizable. The differences of pattern between each position of the measuring spot can be explained by the synchronization between the display temporal responses and the shutter-glasses temporal response. As it 
Lab 1 - Top-left position - Left-eye

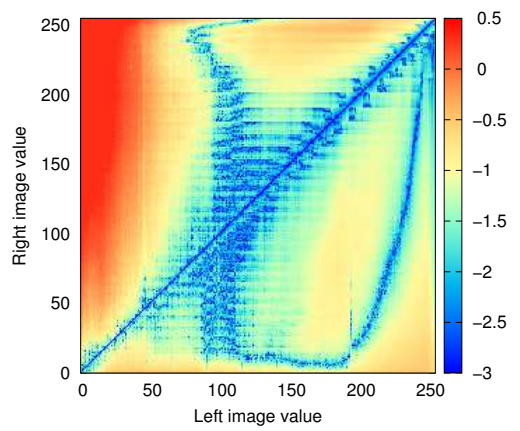

Lab 2 - Top-left position - Left-eye

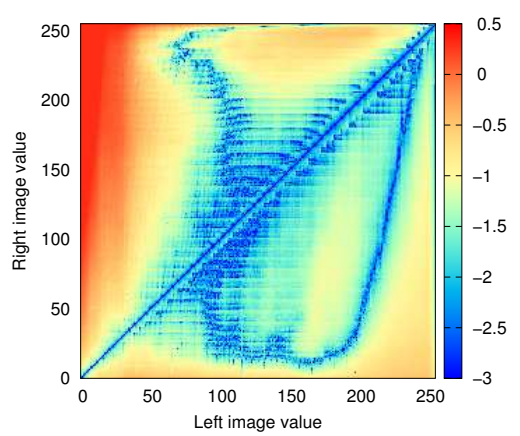

Lab 3 - Top-left position - Left-eye

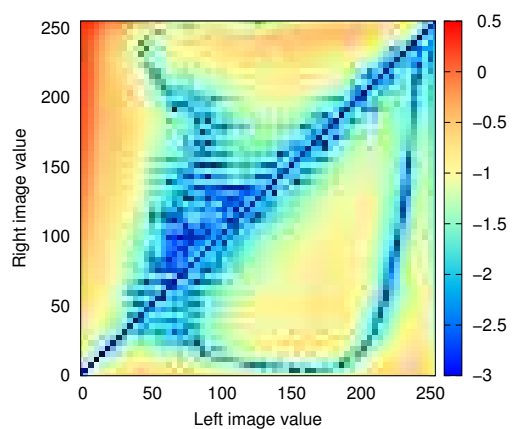

Lab 1 - Center position - Left-eye

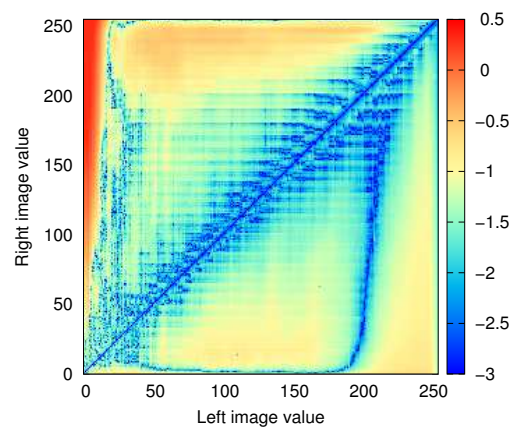

Lab 2 - Center position - Left-eye

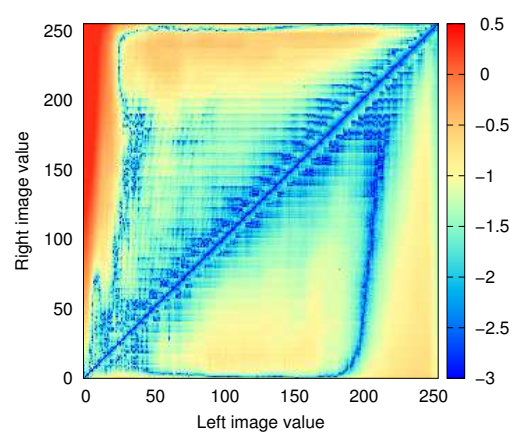

Lab 3 - Center position - Left-eye

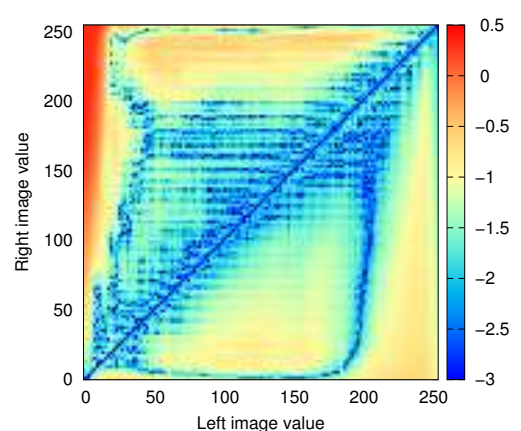

Lab 1 - Bottom-right position - Left-eye

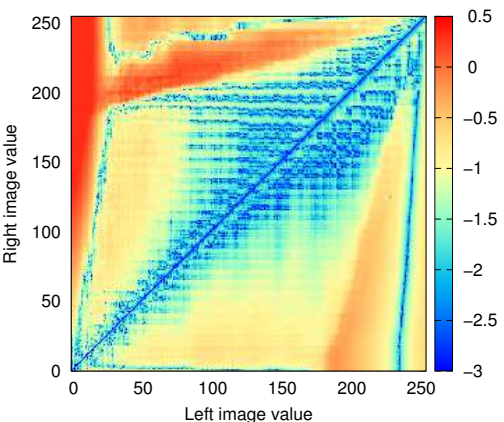

Lab 2 - Bottom-right position - Left-eye

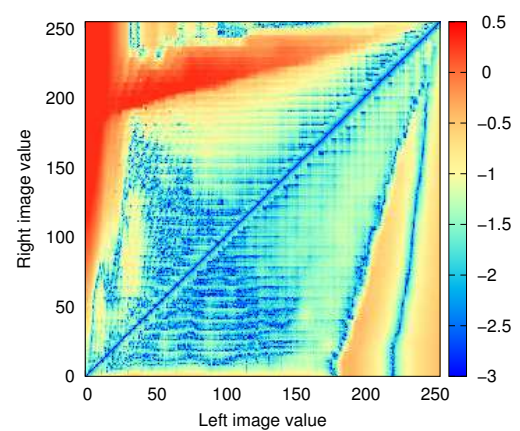

Lab 3 - Bottom-right position - Left-eye

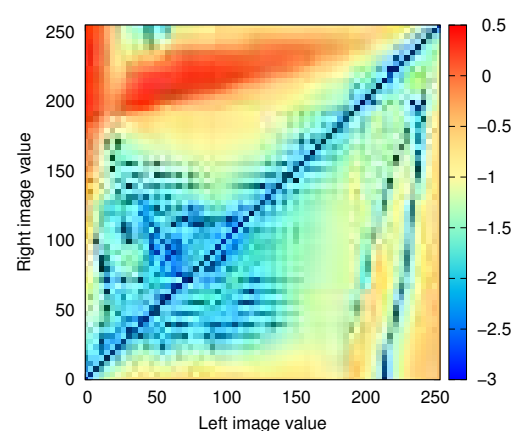

Figure 10: Crosstalk ratios (logarithmic scale) measured at the left-eye position from the luminance matrices presented in Figure 8. 


\begin{tabular}{|c|c|c|c|c|c|c|}
\cline { 2 - 7 } \multicolumn{1}{c|}{} & \multicolumn{2}{c|}{ Top-left } & \multicolumn{2}{c|}{ Center } & \multicolumn{2}{c|}{ Bottom-right } \\
\cline { 2 - 7 } \multicolumn{1}{c|}{} & Method1 & Method2 & Method1 & Method2 & Method1 & Method2 \\
\hline Max & $83.5 \%$ & $36.7 \%$ & $56.3 \%$ & $38.0 \%$ & $118 \%$ & $199 \%$ \\
\hline Avg. & $6.85 \%$ & $7.24 \%$ & $6.45 \%$ & $6.26 \%$ & $9.78 \%$ & $14.6 \%$ \\
\hline StDev. & $7.16 \%$ & $6.77 \%$ & $5.95 \%$ & $7.58 \%$ & $15.4 \%$ & $29.1 \%$ \\
\hline
\end{tabular}

Table 2: Comparison of maximum and average values of crosstalk ratio for both measurement methods in Lab3. These statistics have been computed after having excluded combinations for which the pixels value in the measured view was inferior to 50 (see text).

has been observed in Section 3.3.2 (Figure 6), the luminance level reach by display pixels when the shutter-glass is open are not the same for each position and can be very far from the expected value.

Globally, the highest crosstalk values are found for low grey levels on the measured view and high grey levels in the other. This is not surprising since in that case the reference luminance is very low. This result is in accordance with a previous study, ${ }^{19}$ however one should keep in mind that luminance measurements below 1 $\mathrm{cd} / \mathrm{m}^{2}$ are subject to caution due to the accuracy of luminance-meters.

\section{DISCUSSION}

It is clear from the results presented in that paper that the crosstalk ratio of time-sequential stereoscopic LCDs cannot be evaluated with only one measurement condition. The complexity of the variation of crosstalk ratio across the many combinations of right and left pixels values necessitate to measure crosstalk with a sufficient number of grey-to-grey combinations. The use of $20 \times 20$ grey levels seems to be a minimum to picture crosstalk patterns with a sufficient precision.

Table 2 presents the maximum and average values of crosstalk measured with each measurement method. In order to compare a more coherent range of values, right-left combinations for which the grey level in the measured view was below 50 have been excluded (this corresponds roughly to combinations for which the luminance in the measured view is below $1 \mathrm{~cd} / \mathrm{m}^{2}$ ). It can be observed that the average values are comparable from one method to the other despite of the very high standard deviation. The same tendency can be observed with both methods: the lowest average crosstalk ratio is obtained in the center (around 6\%), then the values obtained in the top-left position are slightly larger, and finally average values in the bottom-right corner are significantly higher.

The main difference between both methods resides in the pattern of the crosstalk matrices: if similar "ridges" and "valleys" can be observed on matrices obtained with both methods, they seem to not be exactly at the same place. This might be due to differences in the conversion into luminance values. The raw data obtained from measurements through the glasses were significantly lower than the raw data obtained direct measurements on the display, and this difference in the data range can have affected the coherence of the conversion into luminance values. Another difference between both measurement method is due to the noise present in the luminance values measured with the first measurement method. Indeed, it has been observed that the measurements performed through the glasses were a bit more noisy than the measurements performed directly in contact with the display. This can be due to the measurement set-up itself which was more complex and sensitive in the former case. Even if this noise itself is not so important, it can be responsible for large variations in crosstalk ratio.

Table 3 gives the same statistics as before, with the same limitations, for crosstalk measured with the second method in each of the three labs. Again, the same tendency is observed: average crosstalk is around $6 \%$ in the center of the screen, slightly higher in the top-left corner (except for crosstalk values measured in Lab2 which are found slightly lower in this corner), and twice higher (around 13\%) in the bottom-right corner.

When comparing crosstalk matrices obtained from one lab to another (Figure 10), it is observed that the similarity of the matrices obtained for each position is quite significant and that the patterns of crosstalk matrices for each measuring positions is strongly recognizable. This is certainly one of the main conclusions of this work: crosstalk variations due to differing measurement positions on the screen are more important than crosstalk variations due to differing instruments and differing set-ups in the three laboratories. This conclusion is confirmed 


\begin{tabular}{|c|c|c|c|c|c|c|c|c|c|}
\cline { 2 - 9 } \multicolumn{1}{c|}{} & \multicolumn{3}{c|}{ Top-left } & \multicolumn{3}{c|}{ Center } & \multicolumn{3}{c|}{ Bottom-right } \\
\cline { 2 - 10 } \multicolumn{1}{c|}{} & Lab1 & Lab2 & Lab3 & Lab1 & Lab2 & Lab3 & Lab1 & Lab2 & Lab3 \\
\hline Max & $137 \%$ & $234 \%$ & $36.6 \%$ & $34.5 \%$ & $36.7 \%$ & $33.6 \%$ & $186 \%$ & $358 \%$ & $214 \%$ \\
\hline Avg. & $7.46 \%$ & $6.31 \%$ & $6.68 \%$ & $6.09 \%$ & $6.84 \%$ & $5.84 \%$ & $13.2 \%$ & $13.6 \%$ & $16.0 \%$ \\
\hline StDev. & $7.93 \%$ & $7.16 \%$ & $6.47 \%$ & $6.03 \%$ & $6.60 \%$ & $5.84 \%$ & $21.1 \%$ & $29.5 \%$ & $31.0 \%$ \\
\hline
\end{tabular}

(a) Left-eye position

\begin{tabular}{|c|c|c|c|c|c|c|c|c|c|}
\cline { 2 - 9 } \multicolumn{1}{c|}{} & \multicolumn{3}{c|}{ Top-left } & \multicolumn{3}{c|}{ Center } & \multicolumn{3}{c|}{ Bottom-right } \\
\cline { 2 - 10 } \multicolumn{1}{c|}{} & Lab1 & Lab2 & Lab3 & Lab1 & Lab2 & Lab3 & Lab1 & Lab2 & Lab3 \\
\hline Max & $156 \%$ & $105 \%$ & $36.7 \%$ & $29.7 \%$ & $35.2 \%$ & $38.0 \%$ & $154 \%$ & $232 \%$ & $199 \%$ \\
\hline Avg. & $8.59 \%$ & $6.05 \%$ & $7.24 \%$ & $5.39 \%$ & $6.81 \%$ & $6.26 \%$ & $13.2 \%$ & $12.6 \%$ & $14.6 \%$ \\
\hline StDev. & $9.32 \%$ & $7.20 \%$ & $6.77 \%$ & $5.63 \%$ & $6.66 \%$ & $7.58 \%$ & $18.6 \%$ & $26.8 \%$ & $29.1 \%$ \\
\hline
\end{tabular}

(b) Right-eye position

Table 3: Maximum and average crosstalk ratio measured with the second method. These statistics have been computed after having excluded combinations for which the pixels value in the measured view was inferior to 50 (see text).

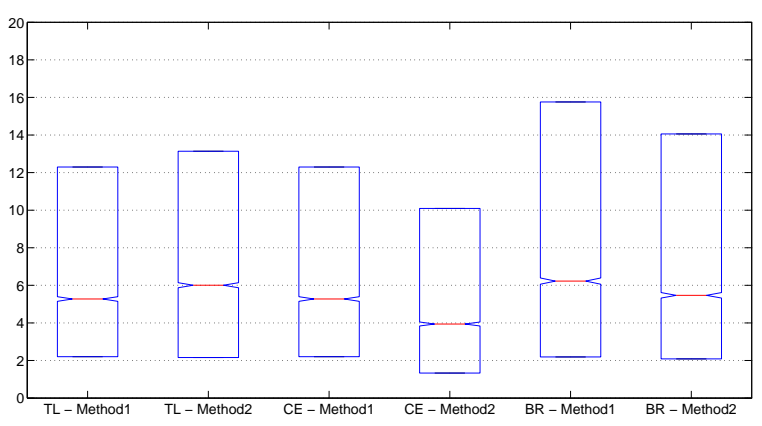

(a)

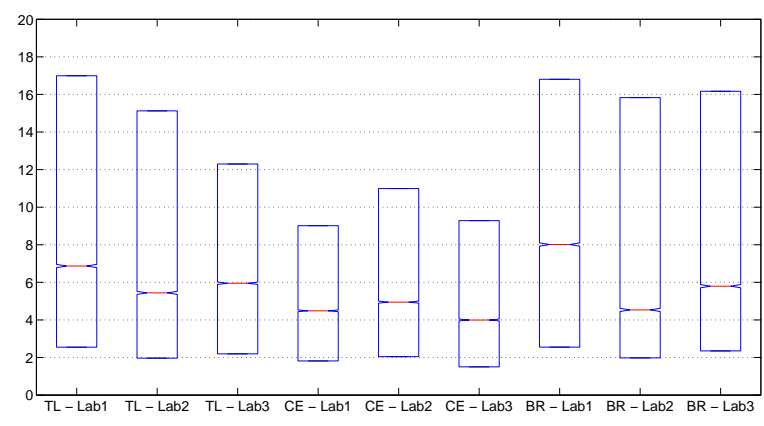

(b)

Figure 11: Comparison of the distributions of crosstalk ratios (values are in percentage). (a) Comparison between both methods for measurements through right glass performed in Lab3. (b) Comparison between laboratories for measurements through the left glass performed with the second method. The red line indicates the median value, the notch indicates the $95 \%$ confidence interval, the blue box delimits the 25 th and 75 th percentiles.

by the study of crosstalk distribution for each condition. Figure 11a compares the distribution of crosstalk ratios measured from the right-eye position with the two different measurement methods, for the three different positions on the screen. Figure 11b presents the distribution of crosstalk values measured from the left-eye position with the second method, in the three labs and for the three different positions on the screen.

\section{CONCLUSION}

In this paper, crosstalk in time-sequential stereoscopic LCDs with active shutter-glasses has been investigated. Two different measurement methods using high sample-rate luminance instruments have been carried out. The first one consisted in measuring luminance directly through the shutter-glasses, while the second one proposed to use the temporal characterization of shutter-glasses transmittance in order to simulate luminance observed through the shutter-glass from the temporal responses of the display. These measurements have been performed in three different laboratories, with three different samples of the same display model. The whole right-to-left combinations have been tested, at three different measuring spots on the screen.

Results showed that the crosstalk ratio is varying according to a pattern which is hardly predictable. This is mainly due to the failure of the display to reach a correct luminance level within the short frame period 
when alternating between different right and left pixels values. Furthermore, significant differences have been found between different positions on the screen. This variability is due to the unequal synchronization between shutter-glasses and display for these positions. This non-linear and non-monotonic behaviour does not permit to describe the crosstalk of a time-sequential stereoscopic LCD with measurements taking into consideration only few conditions. From the crosstalk matrices which have been measured in this study, a minimum of $20 \times 20$ grey levels seems to be necessary to picture a precise enough map of the crosstalk variations at one position of the screen.

This measurement campaign also permitted to show that crosstalk estimates obtained with the second method (which involves a simulation of the shutter-glasses) were quite similar to those obtained with the classical method. This is an important result since the proposed method only requires some close-contact measurements of the display which are usually easier to carry out and less subject to variations. Indeed, crosstalk measurements performed in three different laboratories with this simulation method gave very similar results. In other words, the influence of the right-left combination and the influence of the position of the measuring spot have been found much more important than the influence of the differing instruments and differing set-ups in the three laboratories.

\section{ACKNOWLEDGMENT}

Mid Sweden University's work has been supported by grant 00156702 of the EU European Regional Development Fund, Mellersta Norrland, Sweden, and by grant 00155148 of Länsstyrelsen Västernorrland, Sweden. Acreo's work has been supported by VINNOVA (The Swedish Governmental Agency for Innovation Systems), TCO Development and Intertek Semko. AGH's work has been supported by the Polish Ministry of Science and Higher Education under the European Regional Development Fund, Grant No. POIG.01.01.02-00-045/09-00 Future Internet Engineering.

\section{REFERENCES}

[1] A. Woods, T. Docherty, and R. Koch, "Image distortions in stereoscopic video systems," in Stereoscopic Displays and Applications IV, Proc. of SPIE, Part of ISET/SPIE's International Symposium on Electronic Imaging: Science and Technology 1915, 1993.

[2] W. A. Ijsselsteijn, P. J. Seuntiëns, and L. M. Meesters, Human Factors of 3D Displays, ch. 12, pp. 217-233. John Wiley \& Sons, Ltd, 2006.

[3] M. T. Lambooij, W. A. IJsselsteijn, and I. Heynderickx, "Visual discomfort in stereoscopic displays: A review," in Stereoscopic Displays and Virtual Reality Systems XIV, Proc. of SPIE-ISø3T Electronic Imaging, 6490, 2007.

[4] A. J. Woods, "How are crosstalk and ghosting defined in the stereoscopic literature?," in Stereoscopic Displays and Applications XXII, Proc. of SPIE, Part of IS6TT/SPIE's International Symposium on Electronic Imaging: Science and Technology 7863, 2011.

[5] A. Woods, "Understanding crosstalk in stereoscopic displays," in Three-Dimensional Systems and Applications (3DSA) Conference, (Tokyo, Japan), May 2010. Keynote Presentation.

[6] S. J. Daly, R. T. Held, and D. M. Hoffman, "Perceptual issues in stereoscopic signal processing," IEEE Transactions on Broadcasting 57, pp. 347-361, June 2011.

[7] I. Tsirlin, L. M. Wilcox, and R. S. Allison, "The effect of crosstalk on the perceived depth from disparity and monocular occlusions," IEEE Transactions on Broadcasting 57, pp. 445-453, June 2011.

[8] "Video Quality Expert Group (VQEG)." Website Homepage, 2011. http://www.vqeg.org.

[9] M. A. Weissman and A. J. Woods, "A simple method for measuring crosstalk in stereoscopic displays," in Stereoscopic Displays and Applications XXII, Proc. of SPIE, Part of ISEGT/SPIE's International Symposium on Electronic Imaging: Science and Technology 7863, 2011.

[10] P. Boher, T. Leroux, and V. C. Patton, "Characterization of one time-sequential stereoscopic 3D display part i: Temporal analysis," Journal of Information Display 11, pp. 57-62, June 2010.

[11] P. Boher, T. Leroux, V. C. Patton, and T. Bignon, "Optical characterization of shutter glasses stereoscopic 3D displays," in Stereoscopic Displays and Applications XXII, Proc. of SPIE, Part of ISEST/SPIE's International Symposium on Electronic Imaging: Science and Technology 7863, 2011. 
[12] S. Shestak, D.-S. Kim, and S. Hwang, "Measuring of gray-to-gray crosstalk in LCD based time-sequential stereo display," in SID Symposium Digest of Technical Papers, 41(1), pp. 132-135, 2010.

[13] C.-C. Pan, Y.-R. Lee, K.-F. Huang, and T.-C. Huang, "Cross-talk evaluation of shutter-type stereoscopic 3D display," in SID Symposium Digest of Technical Papers, 41(1), pp. 128-131, 2010.

[14] S.-M. Jung, Y.-B. Lee, H.-J. Park, S.-C. Lee, W.-N. Jeong, J.-K. Shin, and I.-J. Chung, "Improvement of 3-D crosstalk with over-driving method for the active retarder 3-D displays," in SID Symposium Digest of Technical Papers, 41(1), pp. 1264-1267, 2010.

[15] P. Boher, T. Leroux, and T. Bignon, "Grey level crosstalk and temporal synchronization of different shutter glass 3D TVs," in SID Symposium Digest of Technical Papers, 42(1), pp. 164-167, 2011.

[16] J. Bułat, L. Janowski, D. Juszka, M. Sochay, M. Grega, and Z. Papir, "Evaluation of crosstalk metrics for 3D display technologies with respect to temporal luminance analysis," in IEEE Workshop on Multimedia Quality of Experience, 2011.

[17] L. Blondé, J.-J. Sacré, D. Doyen, Q. Huynh-Thu, and C. Thébault, "Diversity and coherence of 3D crosstalk measurements," in SID Symposium Digest of Technical Papers, 42(1), pp. 804-807, 2011.

[18] D. H. Brainard, "The Psychophysics Toolbox," Spatial Vision 10(4), pp. 433-436, 1997.

[19] M. Barkowsky, S. Tourancheau, K. Brunnström, K. Wang, and B. Andrén, "Crosstalk measurements of shutter glasses 3d displays," in SID Symposium Digest of Technical Papers, SID Symposium Digest of Technical Papers 42(1), pp. 812-815, SID, 2011. 